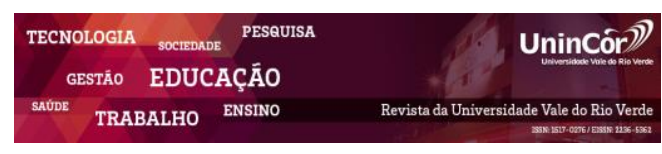

Revista da Universidade Vale do Rio Verde ISSN: 1517-0276 / EISSN: 2236-5362 v. $17 \mid$ n. 1 | Ano 2019

Leandro Carvalho Bassotto Faculdade de Ciências e Tecnologias de Campos Gerais - FACICA bassotto.lc@gmail.com

Luiz Kennedy Cruz Machado Universidade Federal de Lavras - UFLA luizken@yahoo.com.br

Danúbia Teresa Martins Faculdade Pitágoras danubia.tmartins@gmail.com

\section{COMPETITIVIDADE DE UMA PROPRIEDADE DE AGRICULTURA FAMILIAR SOB A ÓTICA DE INDICADORES ECONÔMICOS}

\section{RESUMO}

A pecuária leiteira é um setor de grande importância por contribuir com o desenvolvimento de pequenos agricultores familiares que desenvolvem essa atividade de forma comercial. As vantagens competitivas também são fundamentais para garantir elevados padrões de eficiência no mercado agropecuário e contribuir com o desenvolvimento da cadeia produtiva do leite. Esse estudo tem por objetivo analisar o desempenho econômico de uma propriedade com mão de obra familiar e identificar se esses resultados podem impactar na competitividade desse estabelecimento rural. Foi realizado um estudo de caso exploratório e qualitativo com abordagem descritiva com o intuito de analisar dados econômicos primários de uma propriedade de agricultura familiar do setor leiteiro entre os anos de 2010 a 2017. Embora os investimentos na atividade tenham sido considerados elevados, a propriedade apresentou índices satisfatórios e permitiu a identificação de oportunidades de crescimento e melhoria para o futuro, uma vez que as perspectivas a partir da análise dos dados apresentaram comportamento evolutivo no decorrer dos anos. Foi possível concluir que os indicadores econômicos são fundamentais para o fortalecimento da cadeia produtiva do leite e que são grandes geradores de vantagens competitivas ao setor.

Palavras-chave: Competitividade. Agricultura familiar. Indicadores de eficiência. Pecuária de leite. Estudo de caso.

\section{COMPETITIVENESS OF A PROPERTY \\ OF FAMILY AGRICULTURE UNDER THE OPTICS OF ECONOMIC INDICATORS}

\begin{abstract}
Dairy farming is a sector of great importance for contributing to the development of small family farmers who carry out this activity commercially. The competitive advantages are also fundamental to guarantee high standards of efficiency in the agricultural market and contribute to the development of the milk production chain. The objective of this study is to analyze the economic performance of a property with a family labor force and to identify if these results can impact the competitiveness of this rural establishment. An exploratory and qualitative case study with a descriptive approach was carried out to analyze the primary economic data of a family farm in the dairy sector from 2010 to 2017. Although the investments in the activity were considered high, satisfactory indexes and allowed the identification of opportunities for growth and improvement for the future, once the perspectives from the analysis of the data
\end{abstract}


presented evolutionary behavior over the years. It was possible to conclude that the economic indicators are fundamental for the strengthening of the productive chain of milk and that are great generators of competitive advantages to the sector.

Keywords: Competitiveness. Family farming. Indicators of efficiency. Livestock of milk. Case study.

Recebido em: 17/08/2018 - Aprovado em: 02/04/2019 - Disponibilizado em: 15/07/2019

\section{INTRODUÇÃO}

A atividade leiteira possui diversas áreas primordiais para a geração de renda. Dentro do contexto gerencial, avaliação econômica e planejamento eficazes garantem que os resultados econômicos possam ser melhor trabalhados, permitindo ao produtor conhecer os lucros e prejuízos do seu negócio ao longo do tempo (HOFFMANN et al., 1992; SABBAG; COSTA, 2015) e assegurando maiores vantagens competitivas.

Competitividade é apresentada por Dalazoana et al. (2017) como um elemento fundamental e que deve ser considerado na gestão dos custos de produção, no qual pode contribuir com o desenvolvimento das empresas agrícolas, melhorando a eficiência do processo produtivo. Em um estudo em propriedades leiteiras, Silva, Pedroso e Lages (2017) concluíram que a competitividade é fundamental para que o desenvolvimento econômico das fazendas possa ser realizado, pois permite compreender que o gerenciamento econômico, quando realizado de forma eficiente, pode contribuir significativamente com diversos aspectos relacionados à competitividade das propriedades leiteiras e com o aumento da fronteira do conhecimento sobre esse ramo de negócio agropecuário.

$\mathrm{Li}$ et al. (2017) sugerem que o conhecimento possui ligação direta com a escala produtiva que pode interferir nos resultados da atividade leiteira e em outros setores, como gestão dos custos, financiamentos e estratégias competitivas. Brizolla et al. (2017) salientam que informações gerenciais ligadas aos custos de produção permitem analisar não somente a viabilidade econômica dos processos produtivos como também informações detalhadas sobre diversos aspectos relacionados a vendas, preços, despesas, depreciações, entre outros.

Não obstante, empresas necessitam gerir seus custos corretamente para se conhecer os riscos inerentes ao negócio (RAMOS et al., 2015). Além disso, permite o desenvolvimento de parâmetros gerenciais que auxiliarão no processo de tomada de decisões (LAUGART, 2015).

Com efeito, considera-se a existência de apenas duas maneiras de se intervir nos resultados econômicos da atividade leiteira: aumentar o preço de venda ou reduzir os custos de produção, seja pela diminuição do custo operacional efetivo (COE) ou pelo aumento da produtividade (FIGUEIREDO et al., 2007). Sistemas que contribuam com o desenvolvimento da gestão de 
custos são fundamentais para melhorar os resultados econômicos da atividade leiteira (SABBAG; COSTA, 2015).

Uma das funções da gestão dos custos é fornecer indicadores que auxiliem na administração do negócio. Esses indicadores (de produtividade, zootécnicos e econômicos) são muito baixos no Brasil, condicionando a pecuária leiteira em uma atividade pouco atrativa (LOPES et al., 2016). Ao realizar estudos sobre tais indicadores em fazendas de pecuária leiteira, Silva et al. (2015) concluíram que a melhoria desses indicadores é capaz de deixar a bovinocultura de leite mais atrativa e lucrativa.

Muitos estudos têm sido conduzidos para a identificação de indicadores econômicos que contribuam efetivamente com a avaliação de sistemas de produção (OLIVEIRA et al., 2016). Porém, diversos autores chamam a atenção para a necessidade de estudos mais regionalizados (OLIVEIRA et al., 2007; CAMILO NETO et al., 2012) para contribuir com a evolução da fronteira do conhecimento nesse ramo de atividade.

Esse estudo justifica-se para contribuir com o desenvolvimento de estudos empíricos sobre análises econômicas na bovinocultura leiteira. Para tal, são consideradas as seguintes hipóteses: (I) os indicadores econômicos podem contribuir com o fortalecimento da cadeia produtiva do leite? (II) A gestão eficiente dos custos pode impactar na competitividade de propriedades leiteiras?

Objetivou-se com esse estudo analisar o desempenho econômico de uma propriedade com mão de obra familiar e identificar se esses resultados podem impactar na competitividade desse estabelecimento rural.

\section{MATERIAL E MÉTODOS}

\subsection{Especificações da Pesquisa e dos Dados}

A pesquisa realizada é de natureza qualitativa, exploratória e descritiva (BRYMAN, 1989; SPECTOR, 2002) e possui a finalidade de contribuir com o melhor entendimento daquilo que se pretende estudar (MALHOTRA, 2001). A análise dos dados de um objeto de estudo de forma individual define essa pesquisa como um estudo de caso (GOODE; HATT, 1979), importante para familiarizar o pesquisador com o tema a que se dispões estudar, fato que permite melhores análises dos dados (VIEIRA, 2002).

Quanto à tipologia dos dados, podem ser classificados como dados primários (GIBBS, 2009) uma vez que tenham sido coletados diretamente na propriedade rural, condição que contribui com a mitigação dos riscos de distorções provenientes do manuseio dos dados. A coleta dos dados ocorreu entre os anos de 2010 e 2017, realizadas por um consultor rural que prestava serviços de consultoria gerencial para a propriedade leiteira analisada.

Após a coleta, os dados foram inseridos em uma planilha do Microsoft Excel, de propriedade da empresa Microsoft S./A., momento em que se realizou o cálculo dos indicadores de eficiência, apresentados no tópico a seguir. Nessa etapa, houve a padronização dos dados, que foram agrupados segundo suas especificidades e utilidades dentro da propriedade rural. 


\subsection{O Objeto de Estudo}

A propriedade está localizada no município de Caldas, na microrregião sul do estado de Minas Gerais. Dispõe de mão de obra contratada para realizar as atividades operacionais ligadas à produção de leite, podendo ser classificada, segundo Nahum e Santos (2018), como agricultura familiar. Com relação à contratação de mão de obra externa, apenas algumas contratações esporádicas em períodos sazonais do ano são realizadas para que ações especificas que eventualmente gerem sobrecarga de trabalho não comprometam os resultados da propriedade leiteira.

A propriedade dispõe de 19 hectares (ha) de terra utilizados para a produção leiteira, conforme mostra a Figura 1.

Figura 1 - Áreas utilizadas para a produção leiteira. Caldas, MG - 2018.

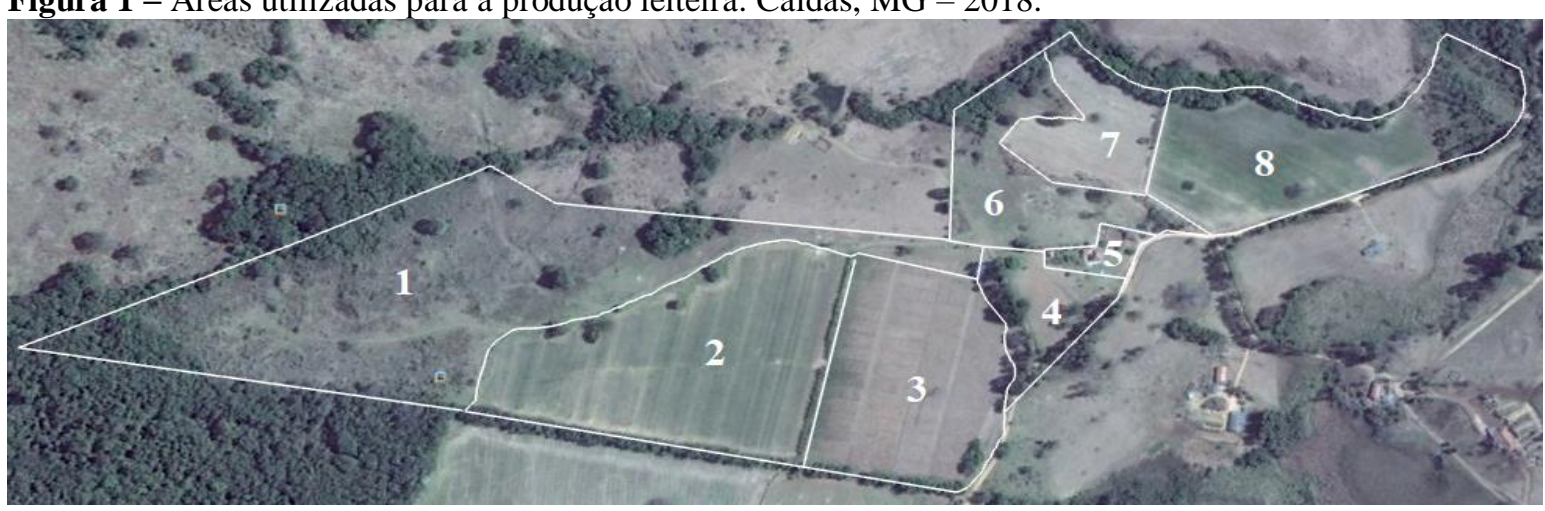

Fonte: Adaptado de Google Maps (2018).

Desse total, 2,5 ha foram comprados em 2015 e 1 ha não pertence à família, sendo arrendado desde períodos anteriores ao início da coleta dos dados, em 2010. As áreas estão divididas (Figura 1) de acordo com suas respectivas utilizações: 1: recria de animais em crescimento (com mais de 1 ano de vida); 2: produção de milho para silagem; 3: produção de pastagens para os animais em produção; 4: recria de animais em crescimento (com menos de 1 ano de vida); 5: benfeitorias e área residencial dos proprietários; 6: área de descanso dos animais; 7: produção de milho para consumo de grãos (arrendado); 8: produção de milho para consumo de grãos (área comprada em 2015).

\subsection{Indicadores de Eficiência}

Foram considerados alguns indicadores econômicos que permitissem analisar aspectos econômicos relacionados da pecuária leiteira. São eles: renda por vaca por ano $(\mathrm{RBa})$ e por dia $(\mathrm{RBd})$, capital investido por vaca do rebanho, custo operacional efetivo (COE) e COE mais investimentos $\left(\mathrm{COE}_{\mathrm{I}}\right)$, taxa de retorno do investimento (ROI) e margem líquida (ML).

Diversos autores concordam que esses indicadores são fundamentais para se analisar o desenvolvimento da atividade leiteira bem como mensurar o grau de desenvolvimento da mesma, quando comparada com outras propriedades (CAMILO NETO et al., 2012; OLIVEIRA et al.; 2016; ASSIS et al., 2017).

Diversos autores contribuíram com a definição dos indicadores (Quadro 1) utilizados nessa pesquisa bem como qual a forma de cálculo 
a ser realizada (CAMILO NETO et al., 2012; MION et al., 2012); SILVA et al., 2015; OLIVEIRA et al., 2016; ASSIS et al., 2017; LOPES et al., 2016).

\section{RESULTADOS E DISCUSSÃO}

A renda bruta por vaca por ano foi maior no ano de 2016 (Tabela 1). Comportamento semelhante pode ser inferido também ao serem comparadas as margens líquidas sobre $\mathrm{COE}$ e $\mathrm{COE}_{\mathrm{I}}$, presentes na Tabela 1. Embora os resultados referentes ao $\mathrm{COE}$ aumentaram a partir de 2016, resultados de $\mathrm{COE}_{\mathrm{I}}$ indicaram que a margem líquida quando se descontou os investimentos realizados permaneceu em conformidade com a média histórica. Pode-se depreender que o $\mathrm{COE}_{\mathrm{I}}$ é mais representativo que as COE para analisar rentabilidade por vaca por ano nesse caso.

Tabela 1 - Índices econômicos anuais expressos em reais/vaca de uma propriedade de agricultura familiar entre os anos de 2010 e 2017. Caldas, MG - 2018.

\begin{tabular}{lcccccccc}
\hline & 2010 & 2011 & 2012 & 2013 & 2014 & 2015 & 2016 & 2017 \\
\hline Renda bruta-RB & $2.255,20$ & $3.263,88$ & $3.413,47$ & $4.320,58$ & $5.211,01$ & $5.074,68$ & $8.283,60$ & $6.835,02$ \\
$\mathrm{COE}_{\mathrm{I}}{ }^{2}$ & $1.458,89$ & $1.849,70$ & $2.237,99$ & $2.455,70$ & $3.150,92$ & $3.581,34$ & $4.919,38$ & $4.278,11$ \\
$\mathrm{COE}_{\mathrm{I}}{ }^{2}$ & $1.649,98$ & $2.243,30$ & $2.406,24$ & $2.700,65$ & $3.236,17$ & $3.649,29$ & $6.303,39$ & $4.607,32$ \\
\hline $\mathrm{ML}^{3}$ sobre COE & 796,31 & $1.414,18$ & $1.175,48$ & $1.864,87$ & $2.060,10$ & $1.493,34$ & $3.364,22$ & $2.556,91$ \\
$\mathrm{ML}^{3}$ sobre COE & & & \\
& 605,22 & $1.020,58$ & $1.007,23$ & $1.619,92$ & $1.974,84$ & $1.425,39$ & $1.980,21$ & $2.227,70$ \\
\hline
\end{tabular}

${ }^{1}$ Custo operacional efetivo; ${ }^{2}$ Custo operacional efetivo + investimento; ${ }^{3}$ Margem líquida.

Fonte: Elaborado pelos autores.

Sobre a diferença dos valores de $\mathrm{COE}_{\mathrm{I}} \mathrm{e}$ COE, constatou-se que foram investidos aproximadamente $\mathrm{R} \$ 2.864,31$ por vaca ao longo dos 8 anos, equivalendo a um montante estimado em $\mathrm{R} \$ 54.708,32$ sobre a média de 14,8 vacas em lactação e 4,3 vacas secas dos outo anos, apresentadas na Tabela 1.

Foi possível estimar também o preço médio de venda do leite nos anos estudados permitindo compará-lo com o $\mathrm{COE}_{\mathrm{I}}$ (em litros de leite) no período. Observou-se que os preços de venda recebidos pelo leite comercializado foram inferiores ao $\mathrm{COE}_{\mathrm{I}}$ nos três primeiros anos da série histórica. Contudo, entre os anos de 2013 e 2017, os resultados se consolidaram em patamares superiores, contribuindo com a melhor viabilidade da atividade leiteira. A tendência exponencial, indicada na Figura 2 sugere também que a média de preços de venda do leite mantém ritmo crescente enquanto que a linha de $\mathrm{COE}_{\mathrm{I}}$ possui crescimento menos acentuado nos anos analisados. 
Figura 2 - Tendência dos preços de venda custos operacionais efetivo por litro da atividade leiteira entre os anos de 2010 e 2017. Caldas, MG - 2018.

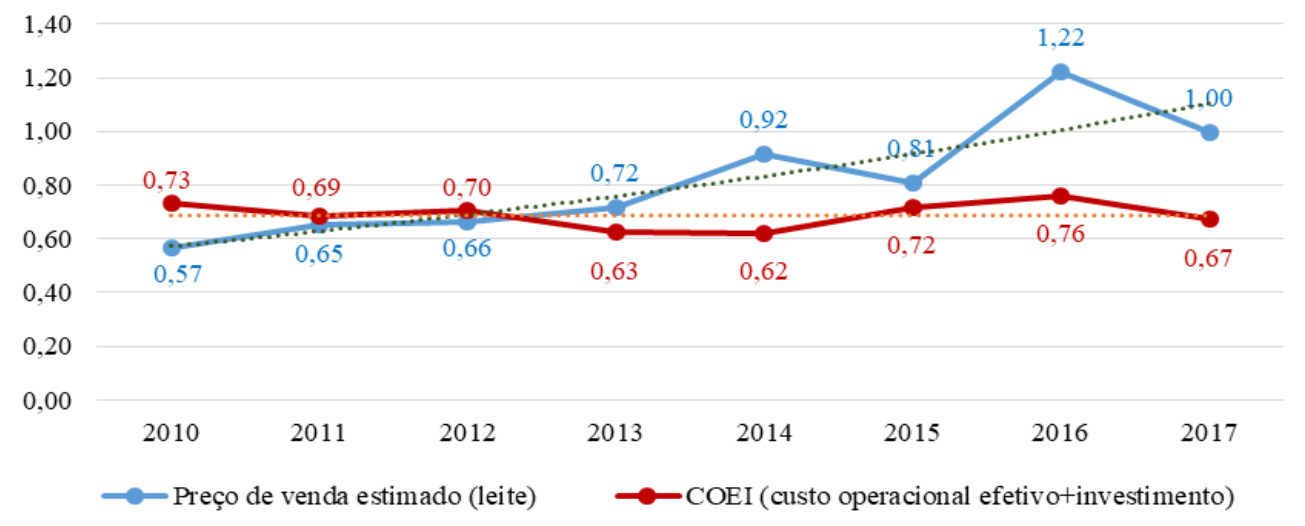

Fonte: Elaborado pelos autores.

A tendência dos preços de venda e $\mathrm{COE}_{\mathrm{I}}$ apresentados na Figura 2 inferem que as perspectivas para o desenvolvimento econômico da propriedade em estudo são favoráveis. A esse respeito, Lhermie et al. (2018) acrescentam que conhecer esses indicadores (e suas tendências de comportamento) são de fundamental importância para a atividade leiteira por contribuir com as práticas de gestão eficiente dos custos (ASSIS et al., 2017).

Ao analisar o capital investido na atividade leiteira, distribuído para cada animal adulto em produção, observou-se forte elevação patrimonial no decorrer dos anos, visto que em 2010, existiam $R \$ 11.742,52$ investidos por vaca e em 2017, esse valor passou para $\mathrm{R} \$ 32.444,67$, alta de $176,3 \%$. Esses resultados podem ser considerados elevados, quando comparados com os mesmos índices calculados na pesquisa de Pereira et al. (2016) que analisaram 159 fazendas que indicaram média de $\mathrm{R} \$ 9.905,00$ investidos por vaca adulta. A Figura 3 apresenta os valores anuais investido por vaca na propriedade.

Figura 3 - Capital investido por vaca na atividade leiteira entre 2010 e 2017. Caldas, MG - 2018.

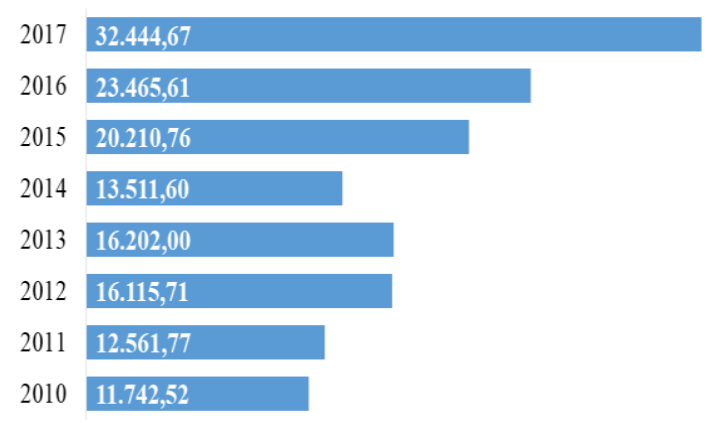

Fonte: Elaborado pelos autores.

Esses índices sugerem a necessidade de elevadas demandas monetárias para a produção leiteira, uma vez que, quando considerado o capital total investido para a produção de leite, percebeu-se certa estabilidade diante de resultados encontrados por outros autores (PEREIRA et al., 2016).

Figura 4 - Capital total investido e taxa de retorno do investimento. Caldas, MG - 2018 .

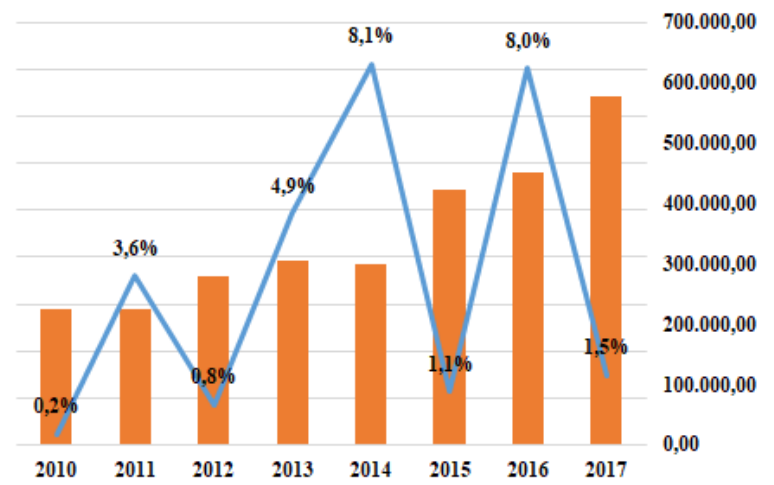

Fonte: Elaborado pelos autores. 
A análise da Figura 4 permitiu constatar forte variação de retorno nos anos analisados. O ROI da atividade foi consideravelmente pequeno nos anos de 2010, 2012, 2015 e 2017. Os anos de 2011 e 2014 apresentaram resultados econômicos satisfatórios por indicarem elevada renda, quando comparada ao capital investido.

Esse fato contribuiu com a elevação do ROI. Contudo, a média do ROI nos anos estudados indicou média anual de 3,54\%, fato que demonstra que o retorno sobre o capital investido na atividade nesses anos pode ser considerado baixo, quando comparado a outras fontes de remuneração do capital investido (BARROS et al., 2018). Para isso, Assis et al. (2017) e Lopes et al. (2016) salientam a importância da escala de produção como mecanismo que contribui com a viabilidade da atividade leiteira.

\section{Análise da Competitividade}

Os dados apresentados na Figura 3 sobre o capital investido (por animal) na atividade leiteira sugerem que a competitividade da propriedade estudada esteja limitada, por demandar elevados recursos monetários investidos que contribuem com a diminuição da taxa de retorno do investimento (ROI), apresentado na Figura 4. Contudo, por não se ter conhecimento aprofundado dos investimentos realizados e dos dados econômicos da propriedade nos anos anteriores ao estudo, torna-se difícil considerar a existência de comportamentos de ascensão ou decesso na atividade, em comparação com os retornos econômicos provenientes dos investimentos.
$\mathrm{O} \mathrm{COE}_{\mathrm{I}}$ foi mais conclusivo que o ROI, quando comparados aos preços de venda da produção (Figura 2) indicaram valorização do produto no mercado e redução dos custos de produção, forte indicativo de competitividade da atividade leiteira. Se as tendências desses indicadores se mantiverem nos próximos anos, é possível que as limitações competitivas encontradas na análise do ROI sejam mitigadas substancialmente.

Esse estudo indicou que o conceito de proposto por Silva, Pedroso e Lages (2017) sobre a importância da competitividade para o desenvolvimento econômico da atividade leiteira é tão real quanto a importância do desenvolvimento econômico para preservar e aumentar a vantagem competitiva das propriedades leiteiras no longo prazo.

\section{CONCLUSÕES}

Os indicadores econômicos considerados nessa pesquisa foram fundamentais para realizar análises mais sólidas e condizentes com a realidade da propriedade. Foi possível identificar a capacidade de fortalecimento da atividade leiteira com o auxílio desses indicadores, fundamentais para análises mais aprofundadas da eficiência de fazendas leiteiras.

Os resultados apresentados indicaram a importância da gestão dos custos para a preservação de elevados padrões de competitividade, assegurando significativa vantagem competitiva às propriedades leiteiras que almejam se manter consolidadas no mercado. Novos estudos serão necessários para estudar 
conceitos relacionados à atratividade econômica e competitividade da atividade leiteira no longo prazo.

\section{REFERÊNCIAS}

ASSIS, L. P. de; VILLELA, S. D. J. LOPES, M. A. L.; SANTOS, R. A. dos; RESENDE, E. S.; SILVESTRE, L. H. A.; SILVA, H. B. F.; MARTINS, P. G. M. de A. Análise econômica e de custos de produção da atividade leiteira durante 10 anos em uma propriedade do Alto Vale do Jequitinhonha. Custos e Agronegócio on line, Recife, v. 13, n. 2, abr./jun. 2017.

BARROS, C. A. P. et al. A contribuição da logística reversa para redução dos custos e do impacto ambiental. Ciências Gerenciais em Foco, v. 4, n. 1, 2018.

BRIZOLLA, M. M. B.; CHIARELLO, T. C.; PLETSCH, C. S.; FASOLIN, L. B.; SILVA, A. da. Sistema de informação sob o enfoque do custeio variável em organizações agrícolas. Custos e Agronegócio online, Recife, v. 13, n. 4, out./dez. 2017.

BRYMAN, A. Research methods and organization studies. 1. ed. London: Unwin Hyman, 1989.

CAMILO NETO, M.; CAMPOS, J. M. de S.; OLIVEIRA, A. S. de; GOMES, S. T. Identification and quantification of benchmarks of Milk productions systems in Minas Gerais. Revista Brasileira de Zootecnia, Viçosa, v. 41, n. 10, p. 2279-2288, 2012.

DALAZOANA, F. M. de L.; BINOTTO, E.; LOPEZ, A. V.; GIMENES, R. M. T. Gestão de custos na produção de fios de algodão. Custos e Agronegócio online, Recife, v. 13, n. 3, jul./set. 2017.

FIGUEIREDO, D. M. de; OLIVEIRA, A. S. de; SALES, M. F. L.; PAULINO, M. F.; VALE, S. M. L. R. do. Análise econômica de quatro estratégias de suplementação para recria e engorda de bovinos em sistema pasto-suplemento. R. Bras. Zootec., v.36, n.5, p.1443-1453, 2007.

GIBBS, G. Análise de dados qualitativos: coleção pesquisa qualitativa. Porto Alegre: Bookman Editora, 2009.

GOODE W. J.; HATT P. Métodos em pesquisa social. 5 ed. São Paulo: Companhia Editora Nacional, 1979.
GOOGLE MAPS. 2018. Disponível em: < https://www.google.com.br/maps>. Acesso em 30 abr. 2018.

LAUGART, S. L. El registro y control de loscostos de calidad. Unanálisis alternativo, em La fabricación de um producto exportable. Anuario Facultad de Ciencias Económicas y Empresariales, v. 5, p. 149159, 2015.

LHERMIE, G.; TAUER, L. W.; GRÖHN, Y. T. The farm cost of decreasing antimicrobial use in dairy production. PloS one, v. 13, n. 3, p. e0194832, 2018.

LI, B.; LIU, Y.; YANG, D.C..; XU, X. The impact of accounting conservatism on the credit availability of agricultural companies: evidence from China. Custos e Agronegócio online, Recife, v. 13, n. 3, jul./set. 2017.

LOPES, M. A.; REIS, E. M. B.; DEMEU, F. A.; MESQUITA, A. A. M.; ROCHA, A. G. F.; BENEDICTO, G. C. de. Uso de ferramentas de gestão na atividade leiteira: um estudo de caso no sul de Minas Gerais. Rev. Cient. Prod. Anim., v.18, n.1, p.26-44, 2016.

MALHOTRA, N.. K. Pesquisa de marketing: uma orientação aplicada. 3 ed. Porto Alegre: Bookman Editora, 2001.

MARQUES, V. M. REIS, R. P.; SÁFADI, T.; REIS, A. J. dos. Custos e Escala na Pecuária Leiteira: estudo de casos em Minas Gerais. Ciênc. agrotec., Lavras. V.26, n.5, p.1027-1034, set./out. 2002.

MION, T. D.; DAROZ, R. Q.; JORGE, M. J. A.; MORAIS, J. P. G. de; GAMEIRO, A. H. Indicadores Zootécnicos e Econômicos para Pequenas Propriedades Leiteiras que Adotam os Princípios do Projeto Balde Cheio. Informações Econômicas, SP, v. 42, n. 5, set./out. 2012.

NAHUM, J. S.; SANTOS, C. B. dos. Agricultura familiar e dendeicultura no município de Moju, na Amazônia paraense. Cuadernos de Geografía: Revista Colombiana de Geografía, v. 27, n. 1, 2018.

NEVES, A. L. A.; PEREIRA, L. G. R.; SANTOS, R. D. dos; ARAÚJO, G. G. L. de; CARNEIRO, A. V.; MORAES, S. A. M.; SPANIOL, C. M. O.; SANTOS, A. Caracterização dos produtores e dos sistemas de produção de leite no perímetro irrigado de Petrolina/PE. Rev. Bras. Saúde Prod. An., Salavadorv.12, n.1, p.209-223 jan./mar., 2011.

OLIVEIRA, M. C.; CAMPOS, J. M. de S.; OLIVEIRA, A. S. de; FERREIRA, M. de A.; MELO, 
A. A. S. de. Benchmarks for Milk Production Systems in the Pernambuco Agreste Region, Northeastern Brazil. Rev. Caatinga, Mossoró, v. 29, n. 3, p. 725-734, Sept. 2016.

OLIVEIRA, V. S. de; FERREIRA, M. de A.; G., A.; MODESTO, E. C.; LIMA, L. E.; SILVA, F. M. da. Substituição total do milho e parcial do feno do capim-tifton por palma forrageira em dietas para vacas em lactação. Produção, composição do leite e custos com alimentação. Revista Brasileira de Zootecnia, Viçosa, v. 36, n. 4, p. 928-935, 2007.

PEREIRA, M. N.; RESENDE, J. C.; PEREIRA, R. A. N.; SILVA, H. C. M. Indicadores de desempenho de fazendas leiteiras de Minas Gerais. Arq. Bras. Med. Vet. Zootec., Belo Horizonte, v. 68, n. 4, p. 1033-1042, ago. 2016.

RAMOS, Y.; SEGURA, A.; ÁLVAREZ, L. M.; RAMÍREZ, F.; ACEVEDO, J. Evaluación de los riesgos económicos y los costos de la calidad en la actividad ganadera de la CCSF Victoria de Girón, Matanzas, Cuba. Pastos y Forrajes, Matanzas, v. 38 , n. 1 , p. 80-84, mar. 2015.

\section{Leandro Carvalho Bassotto}

Mestre em Sistemas de Produção na Agropecuária pela Universidade José do Rosário Vellano UNIFENAS. Especialista em Pecuária Leiteira com Ênfase em Manejo Intensivo de Pastagens pela Universidade Presidente Antônio Carlos - UNIPAC (2014), graduado em Administração pela Universidade Anhanguera Uniderp (2012), Técnico em Agricultura/Zootecnia pela Escola Agrotécnica Federal de Machado (2006). Professor da Faculdade de Ciências e Tecnologias de Campos Gerais FACICA e do Colégio Comercial Professora Ilma Ambrogi Prado.

\footnotetext{
Luiz Kennedy Cruz Machado

Doutorando em Administração pela Universidade Federal de Lavras. Foi professor adjunto na Faculdade de Ciências e Tecnologias de Campos Gerais e professor substituto da Universidade Federal de Alfenas - UNIFAL-MG, lotado no Instituto de Ciências Sociais Aplicadas - ICSA. Mestre em Administração pela Universidade Federal de Lavras na linha de pesquisa Gestão de Negócios Economia e Mercados. Tutor a distância do curso Administração Pública promovido pelo CEADUFLA. Graduado em Administração pela Universidade Federal de São João Del Rei (UFSJ) em agosto de 2012.
}

RODRIGUES, M. dos S. Estudo da Produção de Leite em bovinos: influência do sexo e da cria. Dissertação (Mestrado em Medicina Veterinária) Universidade de Trás-os-Montes e Alto Douro. Vila Real, 2018.

SABBAG, O. J.; COSTA, S. M. A. L. Análise de custos da produção de leite: aplicação do método de Monte Carlo. Extensão Rural, Santa Maria, v. 22, n. 1, p. 125-145, 2015.

SILVA, R. P.; PEDROSO, L. G.; LAGES, A. M. G. Um estudo da competitividade da cadeia produtiva do leite no Estado de Alagoas: uma análise da produção familiar. Custos e Agronegócio online, Recife, v. 13, n. 3, jul./set. 2017.

SILVA, M. F. da; PEREIRA, J. C.; GOMES, S. T.; NASCIF, C.; GOMES, A. P. Avaliação dos indicadores zootécnicos e econômicos em sistemas de produção de leite. Revista de Política Agrícola, v. 24, n. 1, p. 62-73, 2015.

SPECTOR, N. Manual para a redação de teses, dissertações e projetos de pesquisa. 2 . ed. Guanabara Koogan, Rio de Janeiro, 2002.

Danúbia Teresa Martins

Graduanda no curso de Engenharia da Produção da Faculdade Pitágoras. Conclusão em 2018. 